Medizinische Genetik, Universitat Zurich, Ramistrasse 74, 8001 Zurich, Sweitzerland).

COMMENT. Wolf-Hirschhorn syndrome (WHS) is characterized by severe growth and psychomotor retardation, microcephaly, dysmorphic triangular facies ("Greek helmet" appearance), skeletal and cardiac defects. Pitt-RogersDanks syndrome (PRDS) shares features like microcephaly, growth and mental retardation, but is less severe than WHS, without skeletal and cardiac anomalies. Both syndromes are caused by $4 \mathrm{p}$ deletions. Seizures occur in the majority of patients reported, and the EEG findings appear to be characteristic and common to both syndromes, and similar to those in Angelman's syndrome (AS). Periodic bursts of 2-3 Hz high-voltage slow waves with spike wave activity are typically biparietal and associated with myoclonic jerks in sleep in WHS and PRDS, and are usually bifrontal or generalized in AS. Most cases of AS are caused by deletions on chromosome 15q11-13, involving GABA receptor genes. Other GABA receptor genes have been mapped to chromosome 4p. Electroclinical and genetic similarities in WHS and AS suggest a common epileptic mechanism involving GABA pathways. The EEG might be of value in early diagnosis of these syndromes.

\title{
TOPIRAMATE-INDUCED METABOLIC ACIDOSIS IN EPILEPSY
}

Serum bicarbonate (HCO3) levels were measured before, during, and after discontinuing topiramate (TPM) as adjunctive therapy for medically refractory epilepsy in 30 children treated at Children's Hospital, Boston, MA. TPM dose varied from 50 to $650 \mathrm{mg} /$ day, and 2 to $32 \mathrm{mg} / \mathrm{kg} /$ day. Larger doses resulted in higher TPM levels and greater likelihood of low HCO3 levels. Two-thirds had a greater than $10 \%$ decrease in HCO3 levels; the reduction was by 8 and $10 \mathrm{mEq} / \mathrm{L}$ in 2 cases. None was symptomatic. TPM was discontinued in 7 because of ineffectiveness, and in 2 with anorexia. The serum HCO3 returned to pretreatment levels. Monitoring HCO3 levels may be indicated, especially in patients predisposed to acidosis. (Takeoka M, Holmes GL, Thiele E et al. Topiramate and metabolic acidosis in pediatric epilepsy. Epilepsia March 2001;42:387-392). (Reprints: Dr M Takeoka, Child Neurology Service, Department of Pediatrics, School of Medicine, Keio University, 35 Shinanomachi, Shinjuku-ku, Tokyo 160-8582, Japan).

COMMENT. Topiramate is a carbonic anhydrase inhibitor, and a decrease in serum $\mathrm{HCO} 3$ levels with metabolic acidosis may be expected. This study has documented the generally asymptomatic association of lowered HCO3 levels in children treated with adjunctive topiramate therapy for refractory epilepsy. Various mechanisms of action have been proposed for TPM. The carbonic anhydrase inhibitory activity is generally described as weak, but is obviously sufficient to lower HCO3 levels in a substantial proportion of patients treated.

The mechanism of the anticonvulsant action of acetazoleamide, a potent carbonic anhydrase inhibitor, was first demonstrated in 1954 in animal studies at the Department of Pharmacology, University of Utah (Millichap JG, Woodbury DM, Goodman IS. LPharmacol \& Exper Therap 1955;115:251-258; and Proceedings of the American Epilepsy Society meeting, New York, Dec 1954). The anticonvulsant effect is related to the direct inhibition of carbonic anhydrase in the brain, and is independent of the metabolic acidosis which results from inhibition of the enzyme in the kidney. The anticonvulsant effect is not abolished in nephrectomized animals. Metabolic acidosis secondary to acetazolamide (or topiramate) has a very weak anticonvulsant effect, as demonstrated by studies with ammonium chloride in animals tested with electroshock seizures and in the clinic (Millichap JG. Anticonvulsant action of Diamox in children. Neurology 1956;6:552-559). A supplement of bicarbonate, as proposed by Takeoka and 
associates, in some children treated with TPM should not appreciably alter the anticonvulsant effect.

\section{CYTOKINES AND FEBRILE CONVULSIONS}

The production of pro-inflammatory cytokines (IL-1B, IL-6, and TNF-a), and anti-inflammatory cytokine IL-10 by peripheral blood mononuclear cells (PBMC) from 13 children 2 weeks after a febrile convulsion and in 11 without a history of febrile convulsion, was studied at the C Schneider Children's Medical Center, Rabin Medical Center, and Sackler School of Medicine, Tel Aviv University, Israel. Both groups showed an increase in all types of cytokine production, but the secretion of IL-6 and IL-10 in response to lipopolysaccharide stimulation of PBMC was greater in those with a history of febrile convulsions. (Straussberg R, Amir J, Harel L et al. Pro- and anti-inflammatory cytokines in children with febrile convulsions. Pediatr Neurol 2001;24:49-53). (Respond: Dr Bessler PhD, Laboratory for Immunology and Hematology, Rabin Medical Center, Golda Campus, Petah Tiqva, Israel).

COMMENT. Cytokine production by PBMCs of children with a history of febrile seizures at steady state differs from those without convulsions. Infection stimulates immune cells to produce pro-inflammatory cytokines, that can affect brain function and result in seizures. IL-10 inhibits the production of proinflammatory cytokines and has an antipyretic effect. The increased production of IL-10 in the above patients with seizures may be a defense mechanism. The authors comment that cytokine profiles of patients with febrile convulsions and their families may be worthwhile.

\section{HEADACHE DISORDERS}

\section{ACUTE HEADACHE AS AN EMERGENCY PRESENTATION}

A consecutive series of 150 children (aged 2 to 18 years) who presented to an emergency department with abrupt onset of severe headache were evaluated prospectively at the Children's Hospital of the King's Daughters, East Virginia Medical School, Norfork. Causes of acute headache included upper respiratory tract infection with fever (57\%), migraine (18\%), viral meningitis (9\%), posterior fossa tumor (2.6\%), ventriculoperitoneal shunt malfunction (2\%), epileptic seizure with postictal headache (1.3\%), concussion (1.3\%), intracranial hemorrhage (1.3\%), and undetermined (7\%). The URI was viral in $39 \%$, sinusitis in $9 \%$, and streptococcal in $9 \%$. Occipital location and an inability to describe the quality of pain were symptoms significantly associated with serious underlying causes. Pain intensity was not a discriminating factor. Normal activity levels were indicative of a URI-related cause and against serious pathology. Patients with migraine, URI, post-traumatic, and postictal headache had normal neurologic exams. Patients with brain tumor or hemorrhage had obvious neurologic signs, including ataxia, hemiparesis, abnormal eye movements, and papilledema. (Lewis DW, Oureshi F. Acute headache in children and adolescents presenting to the emergency department. Headache March 2001;40:200-203). (Respond: Dr Donald W Lewis, Child and Adolescent Neurology, 850 Southampton Avenue, Norfolk, VA 23510).

COMMENT. The most common cause of abrupt onset of severe headache in children is an upper respiratory tract infection with fever, sinusitis, or migraine. These children may maintain normal activities, the headache is not occipital in location, and the neurologic exam is normal. An occipital headache associated with abnormal neurologic signs is indicative of a serious structural pathology. 